1 Title: Host derived molecules as novel Chagas disease biomarkers: hypercoagulability markers in plasma.

3 Authors: Julio Alonso-Padilla ${ }^{1}$, Dolors Tassies ${ }^{2}$, Nuria Cortes-Serra ${ }^{1}$, Joaquim Gascon ${ }^{1}$, Joan-Carles

4 Reverter², María-Jesús Pinazo ${ }^{1 *}$

6 Affiliations:

$7 \quad{ }^{1}$ Barcelona Institute for Global Health (ISGlobal), Hospital Clínic - Universitat de Barcelona, Barcelona, 8 Spain.

$9 \quad{ }^{2}$ Hemotherapy and Hemostasis Department, Hospital Clínic, Barcelona, Spain.

11 Corresponding author: mariajesus.pinazo@isglobal.org

13 i. Running Head: Host-derived biomarkers for Chagas disease diagnosis.

\title{
ii. Summary/Abstract
}

The most severe clinical symptomatology of Chagas disease affects $\sim 30 \%$ of those chronically

17 infected with the Trypanosoma cruzi parasite. The pathogenic mechanisms that lead to life18 threatening heart and gut tissue disruptions occur "silently" for a longtime in a majority of cases. As a 19 result, despite there are several serological and molecular methods available to diagnose the infection

20 in its acute and chronic stages, diagnosis is often achieved only after the onset of clinical symptoms in 21 the chronic phase of the disease. Furthermore, although there are two drugs to treat it, the assessment 22 of their performance is impractical with current parasite-derived diagnostics and therapeutic efficacy 23 cannot be acknowledged in a timely manner.

24 In this chapter we present two procedures to measure host derived molecules as surrogates of 25 therapeutic response against chronic T. cruzi infection. Their outputs relate to the generation and 
activity of thrombin, a major component of the blood coagulation cascade. This is due to the fact that after treatment with benznidazole.

\section{2}

\section{1. Hypercoagulability markers in plasma: introduction and protocols}

\section{$34 \quad 1.1$ Introduction}

Chagas disease is a complex entity in terms of pathobiology, and complex is the life cycle of its

36 etiological agent: the protozoan parasite T. cruzi. It involves insect and mammalian infective forms,

37 which in the human host are capable of infecting several host cell types and tissues to propagate the infection [1]. During its acute phase there is a strong immunological response as the presence of T. cruzi produces an inflammatory reaction and the secretion of pro-inflammatory cytokines, as well as parasitespecific polyclonal antibodies and activation of effector T cells [2, 3]. Despite this response, the parasite

41 is well-dotted with anti-immune mechanisms and manages to persist leading to a chronic infectious state

42 without causing any overt symptomatology for years. This "silent” clinical feature of Chagas disease 43 largely hinders the clinical diagnosis of the infection before the onset of more severe symptoms [4]. These 44 will affect $\sim 30 \%$ of those chronically infected, who will suffer from damage at the heart and/or at the 45 esophagus and colon. It is when tissue disruption occurs that several unspecific inflammatory mediators 46 appear altered. Before reaching this point, it would be of major relevance to have a way of ascertaining 47 the disease prognosis, including the evaluation of the administration of benznidazole (BNZ) or nifurtimox 48 (NFX) treatments. This would mean a huge advantage for the daily clinical management of the disease, as 49 well as for the clinical testing of any new drug regimens or novel anti-parasitic compounds. 50 Unfortunately, such tool is still missing. 
At present, there are serological and molecular diagnostics based on the respective detection of

52 anti-T. cruzi immunoglobulins or parasite-derived DNA sequences $[5,6]$. They provide valuable

53 information on the infection status, but cannot inform on the clinical prognosis of the disease; and despite

54 recent advancements [7], they cannot yet be used to address treatment efficacy (or spontaneous cure)

55 either. This is because a positive serological result takes many years to become negative making serology

56 impractical for response-to-treatment assessment. Whereas regarding highly sensitive molecular-based

57 techniques, although a positive detection certainly indicates there are circulating parasites, a negative

58 result cannot rule out a latent very low parasitemia or the presence of tissue hidden forms that could

59 relapse later on. Thereby, in view of the absence yet of markers to address disease prognosis and/or

60 therapeutic response in the parasite, host-derived markers have also become a source of interest.

61 Even though host-parasite interactions and their role in the progression of the disease are still not

62 well known, several host biomarkers of T. cruzi infection have been identified during the last decade [8].

63 Moreover, their use as diagnostics to evaluate therapeutic response has been investigated [8]. In this

64 regard, there are three main groups of host-derived molecules: (i) immunological markers (cytokines and

65 surface markers) elicited by the host cellular response to the infection [9, 10]; (ii) biochemical

66 biomarkers, such as hypercoagulability markers, fragments of apolipoprotein A1 (ApoA1), tumor necrosis

67 factor (TNF) or transforming growth factor beta (TGF $\beta$ ) [11-13]; and (iii) inflammatory markers of

68 cardiac damage (e.g. type-B natriuretic peptide (BNP) or highly-sensitive protein C), which have been

69 perhaps the most studied, and shown to be not very good to follow disease progression [14].

$70 \quad$ It has been previously described that a hypercoagulability state can appear in people with $T$. cruzi

71 infection $[11,15,16]$. In the context of an infectious disease, this hypercoagulability could be due to three

72 processes: (a) dysregulation of immunothrombosis [17]; (b) platelet adhesion events driven by a chronic

73 inflammation state [18]; and (c) vasculitis caused by the chronic infection [19]. Altogether they can lead

74 towards increased levels of pro-inflammatory cytokines, and therefore perpetuate the risk of thrombotic

75 accidents, which is one of the main causes of pathophysiology in Chagas disease. 
Amongst the different blood coagulation markers involved in the aforementioned processes, two were significantly elevated in Chagas disease patients in comparison to controls and could thereof be used as biomarkers of $T$. cruzi infection diagnosis and response-to-treatment assessment [16] (Figure 1). The hypercoagulability markers prothrombin fragment $1+2(\mathrm{~F} 1+2)$ and endogenous thrombin potential (ETP)

80 were abnormally expressed in a high percentage of patients with chronic T.cruzi infection before

81 treatment (77\% and 50\%, respectively). Shortly after BNZ treatment both returned to, and remained at 82 normal levels in 76\% and 96\% of patients, respectively [16]. The performance of F1+2 and ETP fulfilled 83 the Target Product Profile (TPP) defined for chronic Chagas disease response-to-treatment biomarkers [8, $8416]$.

85 It cannot be disregarded that both hypercoagulability biomarkers (F1+2 and ETP) presented with 86 normal values in a percentage of $T$. cruzi-infected people, which may limit their usefulness as universal 87 biomarkers [16]. Another big limitation is that current procedures to measure them share a common 88 requirement for high-technological equipment and highly-trained technical personnel to run it. This is a 89 setback for their application in many ill-equipped laboratories from Chagas disease endemic regions at the 90 moment. Indeed there is yet a lot of work ahead, and more resources and attention should be placed on the 91 matter.

92 In this chapter, we describe the rational and protocol procedures to implement the detection of 93 presently most promising host-derived biomarkers for Chagas disease response-to-treatment assessment.

94 These are the hypercoagulability biomarkers F1+2 Enzyme-Linked Immunosorbent Assay (Protocol 1), 95 and Endogenous Thrombin Potential (ETP) Kinetics Assay (Protocol 2) [16].

$97 \quad 1.2$ Protocol 1: F1+2 Enzyme-Linked ImmunoSorbent Assay

99 coagulation cascade. Thrombin activation is accompanied by formation of fragment F1+2. Thus, 100 quantification of F1+2 allows tracking the thrombin formation process. By measuring the concentration 
101 of F1+2 in plasma samples it is possible to diagnose prothrombotic states in comparison to plasma control

102 samples. Levels of F1+2 can be detected with an immunochemical assay, such as the Enzygnost F1+2

103 that is commercialized by Siemens Healthcare (reference number OPBD03; Marburg, Germany). This is

104 an enzyme-linked immunosorbent assay (ELISA) based on an F1+2-specific monoclonal antibody (mAb).

105 It is a sandwich ELISA because the antigen to detect $(\mathrm{F} 1+2)$ is first bound by mAb that come attached to

106 the assay wells. In a second incubation step, another antibody, specific against another antigenic

107 determinant of F1+2 is added in to close the sandwich. This latter antibody is conjugated to a peroxidase

108 (POD) enzyme and thus the presence of sandwich complexes can be evaluated with the addition of a

109 chromogenic substrate of the POD reaction. The optical density (OD) value is proportional to the

110 concentration of $\mathrm{F} 1+2$, the higher the absorption the higher is the concentration of $\mathrm{F} 1+2$ in the plasma

111 sample (Figure 2). The concentration of F1+2 in the samples is calculated by comparing the OD of the

112 samples to the calibration curve made with standard specimens.

113

114 1.3 Protocol 2: Endogenous Thrombin Potential (ETP) Kinetics

115 The Endogenous Thrombin Potential (ETP) is a test that reflects the capacity of a sample to 116 generate thrombin considering both formation and inhibition. ETP can be determined measuring the 117 conversion kinetics of a synthetic thrombin substrate. ETP test is performed by means of a commercial 118 assay (i.e. ETP Innovance by Siemens Healthcare) and the required corresponding piece of equipment 119 (i.e. Siemens BCS System). These resources are available in several hospital Hemostasis units.

120 The synthetic thrombin substrate included in the kit contains a chromophore that is released upon 121 its thrombin-mediated catalysis, therefore providing a chromogenic readout of thrombin activity in the 122 plasma samples. The BCS System records absorbance at $405 \mathrm{~nm}$ in a continuum to evaluate the 123 enzymatic kinetics of thrombin activity. BCS can also calculate the test output values. It first corrects with 124 a mathematical algorithm for the thrombin bound to $\alpha_{2}$-macroglobulin so that the corrected curve shows 125 free thrombin kinetics and the end level of this curve correspond to the ETP value. ETP calculations are 
126 automatically performed by the BCS equipment. The area under the corrected curve (AUC) and the

127 curve's peak height $\left(\mathrm{C}_{\max }\right)$ have been shown to be of diagnostic relevance. They can be obtained by an 128 specific additional software (provided by the manufacturer) from the thrombin generation curve, that is 129 the first derivative of the corrected curve.

\section{Materials}

This section is organized to list out all reagents and equipment required to perform the 133 aforementioned protocols.

\section{2.1. F1+2 ELISA (Protocol 1)}

135 1. Preparation of plasma sample specimens.

a. Venous blood samples (see Notes 1 to 3 in Protocol 1).

b. Sodium citrate $3.2 \%$ solution $(0.11 \mathrm{~g} / \mathrm{L})$.

c. Table top centrifuge and rotor capable of 2,500 g centrifugation. 1).

a. Enzygnost anti-F1+2 mouse mAb coated wells in strips format. Concentration of the mAbs may range from 10 to $100 \mu$ g per well depending on the lot.

b. Microtiter assay plates to mount the strips in to perform the test. concentration can range from 2 to $20 \mathrm{mg} / \mathrm{L}$ depending on the lot. bovine serum albumin. concentrations respectively range from $\sim 20$ to $\sim 1,200 \mathrm{pmol} / \mathrm{L}$ (depending on the specific lot) in a Z-fold increase/decrease manner. 
f. Control plasma: lyophilized human plasma with F1+2 value as assigned in the corresponding lot.

g. Sample buffer to dilute samples: Tris-HCl buffer that contains Tween and sodium chloride $(\mathrm{NaCl})$.

h. Washing solution: phosphate buffer containing Tween provided by the manufacturer.

i. Buffer solution for dilution of TMB substrate containing hydrogen peroxide $\left(\mathrm{H}_{2} \mathrm{O}_{2}\right)$ in acetate buffer.

j. Tetramethyl benzidine dihydrochloride (TMB) chromogenic substrate.

k. Stopping solution to stop the POD reaction: sulfuric acid $\left(\mathrm{H}_{2} \mathrm{SO}_{4}\right)$ at $0.25 \mathrm{~mol} / \mathrm{L}$.

3. Materials not provided by the kit.

a. Micro-pipettes of variable volume dispensing capacities (from $50 \mu \mathrm{L}$ to $1 \mathrm{~mL}$ ), and multichannel pipette suitable to dispense from $50 \mu \mathrm{L}$ up to $300 \mu \mathrm{L}$.

167

\subsection{Endogenous Thrombin Potential (ETP) Kinetics (Protocol 2)}

1. Preparation of plasma sample specimens: it is the same as that referred for Protocol 1 (see section 2.1) (see Notes 1 to 3 in Protocol 2).

2. Reagents included in the ETP kit (see Note 4 in Protocol 2).

a. ETP Reagent containing fibrin aggregator inhibitor, chromogenic substrate, salts and stabilizer.

b. Calcium chloride $\left(\mathrm{CaCl}_{2}\right)$ solution at $250 \mathrm{mM}$.

c. ETP Buffer consisting of Tris-HCl $50 \mathrm{mM}$ at pH 7.4. 


\section{Reagents not included in the kit. (See Note 5 in Protocol 2).}

a. Innovin Reagent (Innovin is a recombinant thromboplastin reagent).

b. ETP Standard (ETP Standard can be purchased to the same manufacturer than ETP).

4. Equipment: the hemostasis analyzer for the ETP assay by Siemens Healthcare is the BCS System (see Note 6 in Protocol 2).

\section{Methods}

\subsection{F1+2 ELISA (Protocol 1)}

1. Obtain venous blood mixing 9 parts of blood with 1 part of sodium citrate solution $3.2 \%$ (0.11 $\mathrm{mol} / \mathrm{L})$.

2. Centrifuge the mix for 15 minutes at $2,500 \mathrm{~g}$.

3. Get the upper supernatant plasma, taking care not to carry over platelets as well.

4. Fresh plasma samples can be stored at room temperature (15 to $25^{\circ} \mathrm{C}$ ) for 4 hours or in the fridge ( 2 to $8{ }^{\circ} \mathrm{C}$ ) for 8 hours until use. If longer storage is required, samples can stay frozen below $-60{ }^{\circ} \mathrm{C}$ in cap sealed tubes for up to 6 months (see Notes 2 and 5 in Protocol 1).

5. Following the manufacturer instructions prepare the following solutions: "Washing solution", “Conjugate solution”, and “Chromogen working solution”. All reagents and samples must be at room temperature before starting the procedure.

6. Pick up the number of strips required considering that all determinations (standards 1 to 4 ), control samples and patients samples) must be done in duplicate (ensuring the coefficient of variation $(\mathrm{CV})$ is $\leq 15 \%)$ (see Note 6 in Protocol 1$)$.

7. Add $50 \mu \mathrm{L}$ of Sample buffer into each well. Then add $50 \mu \mathrm{L}$ of standards, controls and samples where corresponding. Agitate the plate thoroughly (e.g. by aspirating and dispensing in well contains a few times with a multi-channel pipette) to mix well the reagents. 
8. Cover the plate with an adhesive foil and incubate for 30 minutes at $37^{\circ} \mathrm{C}$ in a water bath (see Note 7 in Protocol 1).

9. Remove the foil, aspirate the content of the wells and wash $2 \mathrm{X}$ by addition of $300 \mu \mathrm{L}$ of plate on tissue paper.

10. Add in $100 \mu \mathrm{L}$ per well of "Conjugate solution", not dispensing it onto the edge of the well.

11. Cover with foil and incubate for 15 minutes at $37^{\circ} \mathrm{C}$.

12. Remove the foil, aspirate the wells contain and wash $3 X$ as described earlier. Tap the inverted plate a few times against tissue paper to get rid of any “Conjugate solution” remainders.

13. Add in $100 \mu \mathrm{L}$ per well of "Chromogen solution". plate light-protected.

15. Remove the foil and add in $100 \mu \mathrm{L}$ per well of Stopping solution. $\mathrm{nm}$ and $650 \mathrm{~nm}$ wavelengths.

17. To evaluate results calculate mean absorbance values of duplicates. Construct a reference curve with the Standards values ( $X$-axis F1+2 concentration range from 20 to $1200 \mathrm{pmol} / \mathrm{L}$; $Y$ axis reflecting Absorbance at $450 \mathrm{~nm}$ ranging from 0.01 to 3). F1+2 concentrations of controls and samples can be directly extracted from the reference curve using their calculated mean absorbance values. Healthy donor control samples concentrations are expected to fall within an interval of reference values (see Note 8 in Protocol 1). F1+2 values above that reference range can indicate a hypercoagulable state (see Notes 9 and 10 in Protocol 1); whereas values below it can indicate hypocoagulable state. Please, be aware that a series of interfering substances in the samples may interfere with the test output (see Note 11 in Protocol 1). 


\subsection{Endogenous Thrombin Potential (ETP) Kinetics (Protocol 2)}

226 1. Obtain venous blood mixing 9 parts of blood with 1 part of sodium citrate solution $3.2 \%(0.11$ $227 \mathrm{~mol} / \mathrm{L})$

228 2. Centrifuge the mix for 15 minutes at a 2,500 g.

229 3. Get the upper supernatant plasma, taking care not to carry over platelets as well.

230 4. Fresh plasma samples can be stored at room temperature (15 to $25^{\circ} \mathrm{C}$ ) for 4 hours until use. If 231 longer storage is required, freeze them below $-60{ }^{\circ} \mathrm{C}$ in cap sealed tubes.

232 5. Plasma controls for normal ETP range (control negative pool) and pathological ETP range (control 233 positive pool) must be made in advance and stored aliquoted in cap sealed tubes below $-60{ }^{\circ} \mathrm{C}$ until

234 needed. Confidence intervals of these controls should be calculated and established at \pm 2.5 standard 235 deviations from their median value (see Note 7 in Protocol 2). Controls will be treated as samples 236 and included in each run. Please, be aware that a series of interfering substances in the samples may 237 interfere with the test output (see Note 8 in Protocol 2).

238 6. Thrombin formation in the samples and controls is driven by addition of Innovin Reagent 239 (Siemens Healthcare) and calcium chloride.

240 7. The assay runs over 20 minutes during which the thrombin continuous kinetics is displayed by the $241 \quad$ BCS System (Note 9 in Protocol 2).

242 8. The test output is the end value of ETP conversion or the AUC of the kinetics corrected curve 243 since both are proportional. Both parameters are automatically provided by the BCS System installed 244 software (Note 10 in Protocol 2). 
251 1. Improper collection of blood samples or insufficient mixing with citrate solution may lead to falsely elevated F1+2 values. A clean venipuncture is essential. The required minimum draw volume is $2.7 \mathrm{~mL}$ blood in a $3 \mathrm{~mL}$ tube. Do not use the first $2 \mathrm{~mL}$ of blood collected. Mix thoroughly the tube by gentle inversion immediately after venipuncture.

2. Do not refrigerate the whole blood tubes. Prothrombin F1+2 is unstable at room temperature and tubes must be processed and frozen within four hours of sample collection.

3. Sample rejection causes are clotted sample, overfilled or underfilled tube, mislabeled or unlabeled sample.

4. Good performance of the test is guaranteed only if reagents have the same lot number or the required combination of lot numbers is used.

5. It is not possible to measure F1+2 with Enzygnost monoclonal antibody out of samples that have been frozen and thawed repeatedly.

6. Each experiment and each plate used within must include its own standard curve using the provided standard samples 1 to 4 . Besides, the "Control plasma” supplied with the kit has to be tested along with each series of samples. To validate the test, "Control plasma” sample F1+2 concentration must fall within the expected range as determined in the kit lot instructions.

7. Be aware that plate filling must be completed within ten minutes and the incubation time starts when the plate is placed in the water bath at $37^{\circ} \mathrm{C}$.

8. Median value of F1+2 from healthy adults' citrated plasma $(n=137)$ was reported to be 115 $\mathrm{pmol} / \mathrm{L}$ (with $5^{\text {th }}$ to $95^{\text {th }}$ reference range percentile of 69 to $229 \mathrm{pmol} / \mathrm{L}$ ). This reference interval may vary from lab to lab and that is why each laboratory should establish its reference working interval. If any Absorbance values exceed that of the highest standard, such samples will have to be re-tested diluted (maximum 1:20) in Sample buffer. Be aware of the dilution factor to correct results obtained. 
9. Plasma F1+2 levels are increased in patients with renal failure.

276 10. Since Enzygnost F1+2 detection procedure is based on a F1+2 specific monoclonal antibodies, be aware that patients who have received preparations of mouse monoclonal antibodies may yield falsely elevated results.

11. In relation to interfering substances present in the plasma samples, it has been reported that Enzygnost F1+2 can work with levels of: bilirubin up to $60 \mathrm{mg} / \mathrm{dL}$, free hemoglobin up to 600 $\mathrm{mg} / \mathrm{dL}$, lipids up to $3000 \mathrm{mg} / \mathrm{dL}$, and rheumatoid factors up to $197 \mathrm{IU} / \mathrm{mL}$.

\section{Endogenous Thrombin Potential (ETP) Kinetics. Protocol 2}

1. Improper collection of blood samples or insufficient mixing with citrate solution may lead to erroneous ETP values. A clean venipuncture is essential. The required minimum draw volume is

2. Do not refrigerate the whole blood tubes. Tubes for ETP determination must be processed and frozen within four hours of specimen collection.

3. Sample rejection causes are clotted sample, overfilled or underfilled tube, mislabeled or unlabeled sample.

4. We have previously used an ETP kit provided by Dade Behring (reference number OPDS05; Marburg, Germany). Siemens Healthcare now provides a product called ETP Innovance, which is CE-marked and thus useful for research as well as for diagnostic purposes.

5. Innovin Reagent is purchased from Siemens (reference number B4212). Similarly occurs with the ETP Standard (reference number OPDR05).

6. ETP kit can only be used in the BCS System.

7. Repetitive freeze-thaw cycles will damage control samples integrity. Therefore it is encouraged to prepare pools and store them frozen below $-60{ }^{\circ} \mathrm{C}$ in aliquots containing the required operational 
volumes. If the values of the control samples included in the run are outside their predetermined confidence intervals, the controls, the test reagents, and the BCS analyzer must be checked.

302 8. Icteric, hemolytic and lipemic samples may interfere with the procedure.

303 9. BCS System rotor cuvette can run 20 samples in each run. Since measurement takes relatively 304 long time (20 minutes), ETP should be performed in batches since no other test can be made in 305 the BCS whilst ETP is running.

306 10. Current BCS System does have checking algorithms for the ETP assay. If visual inspection of 307 thrombin kinetics curves by operator, plausible kinetics must follow an initial linear rapid rise 308 that then reaches a lag (plateau) phase with a much slower linear rise. ETP Standard Plasma curve 309 can be used for comparison (see Figure 3). Invalid kinetics may show multiple rising phases, or 310 an uneven linear growth at the end of the reaction time. Curves that have falling sections are not valid either and should be repeated.

\section{5. References}

314 1. Maeda FY, Cortez C, Alves RM, Yoshida N (2012) Mammalian cell invasion by closely related 315 Trypanosoma species T. dionisii and T. cruzi. Acta Trop. 121 (2):141-47.

316 2. Grauert MR, Houdayer M, Hontebeyrie-Joskowciz M (1993) Trypanosoma cruzi infection 317 enhances polyreactive antibody response in an acute case of human Chagas’ disease. Clin Exp 318 Immunol. 93 (1):85-92.

319 3. Cardillo F, Voltarelli JC, Reed SG, Silva JS (1996) Regulation of Trypanosoma cruzi infection in 320 mice by gamma interferon and interleukin 10: role of NK cells. Infect. Immun. 64 (1):128-34.

321 4. Gascon J, Pinazo M-J (2015) Chagas disease: from Latin America to the world. Reports Parasitol $322 \quad$ Volume 4:7.

323 5. Schijman AG (2018) Molecular diagnosis of Trypanosoma cruzi. Acta Trop. 1-8-2018.

324 6. Abras A, Gállego M, Llovet T, Tebar S, Herrero M, Berenguer P, Ballart C, Martí C, Muñoz C 

54(6):1566-1572.

327 7. Zrein M, Granjon E, Gueyffier L, et al (2018) A novel antibody surrogate biomarker to monitor 328 parasite persistence in Trypanosoma cruzi-infected patients. PLoS Negl Trop Dis. 12(2):e0006226.

329 8. Pinazo M-J, Thomas MC, Bua J, et al (2014) Biological markers for evaluating therapeutic 330 efficacy in Chagas disease, a systematic review. Expert Rev Anti Infect Ther 12(4):479-496.

331 9. Egüez KE, Alonso-Padilla J, Terán C, Chipana Z, García W, Torrico F, Gascon J, Lozano-Beltran 332 D-F, Pinazo M-J (2017) Rapid diagnostic tests duo as alternative to conventional serological 333 assays for conclusive Chagas disease diagnosis. PLoS Negl Trop Dis. 11(4):e0005501.

334 10. Keating SM, Deng X, Fernandes F, et al (2015) Inflammatory and cardiac biomarkers are

11. Pinazo M-J, Tàssies D, Muñoz J, Fisa R, Posada EDJ, Monteagudo J, Ayala E, Gállego M, Reverter J-C, Gascon J (2011) Hypercoagulability biomarkers in Trypanosoma cruzi-infected patients. Thromb Haemost. 106(4):617-23.

12. Ndao M, Spithill TW, Caffrey R, et al (2010) Identification of novel diagnostic serum biomarkers for chagas’ disease in asymptomatic subjects by mass spectrometric profiling. J Clin Microbiol

342 13. Curvo EO, Ferreira RR, Madeira FS, Alves GF, Chambela MC, Mendes VG, Sangenis LHC, Waghabi MC, Saraiva RM (2018) Correlation of transforming growth factor-beta1 and tumour necrosis factor levels with left ventricular function in Chagas disease. Mem Inst Oswaldo Cruz. 113(4):e170440.

14. Okamoto EE, Sherbuk JE, Clark EH, et al (2014) Biomarkers in Trypanosoma cruzi-infected and uninfected individuals with varying severity of cardiomyopathy in Santa Cruz, Bolivia. PLoS Negl Trop Dis. 8(10):e3227.

15. Herrera R, Diaz E, Perez Aguilar R, Bianchi J, Berman S, Luciardi HL (2005) Prothrombotic state 
in early stages of chronic Chagas’ disease. Its association with thrombotic risk factors. Arch. Cardiol. Mex. 75 Suppl 3 S3:38-48.

352 16. Pinazo M-J, Posada E de J, Izquierdo L, et al (2016) Altered hypercoagulability factors in patients with chronic Chagas disease: potential biomarkers of therapeutic response. PLoS Negl Trop Dis 10(1):e0004269.

17. Engelmann B, Massberg S (2013) Thrombosis as an intravascular effector of innate immunity. Nat Rev Immunol. 13(1):34-45.

18. Schuetz P, Christ-Crain M, Morgenthaler NG, Struck J, Bergmann A, Müller B (2007) Circulating precursor levels of endothelin-1 and adrenomedullin, two endothelium-derived, counteracting substances, in sepsis. Endothel J Endothel Cell Res. 14(6):345-51.

19. Kreutz RP, Tantry US, Bliden KP, Gurbel PA (2007) Inflammatory changes during the "common 


\section{$375 \quad$ Figure captions.}

376

377 Figure 1. Scheme of the blood coagulation cascade. Hypercoagulability biomarkers of the present chapter 378 are highlighted in bold.

379

380 Figure 2. Graph illustrating a typical calibration curve of F1+2.

381

382 Figure 3. Plot of the measured total conversion and the calculated thrombin kinetics of a plasma sample.

$383 \quad Y$-axis shows the level of conversion of the synthetic substrate, whereas time of reaction is represented in 384 the $X$-axis.

385 